\title{
Atomic and Molecular Gas Components in Spiral Galaxies of the Virgo Cluster
}

\author{
A. V. Kasparova* \\ Sternberg Astronomical Institut M. V. Lomonosov Moscow State Univ.
}

\begin{abstract}
Based on two models, we investigate the molecular-to-atomic gas ratio in Virgo cluster galaxies in comparison with field galaxies. We show that the enhanced metallicity for cluster members and the ram pressure stripping of atomic gas from the disk periphery cannot fully explain the observed gas component ratios. The additional environmental factors affecting the interstellar medium and leading to an increase in the molecular gas fraction should be taken into account for cluster galaxies.

Keywords: interstellar gas in galaxies, Virgo cluster.
\end{abstract}

Astronomy Letters, 2012, Vol. 38, No. 2, pp. 63-73.

\section{INTRODUCTION}

Atomic and molecular hydrogen are the main components of the interstellar medium, with the mechanisms of the transition from one gas state to the other being known poorly and being the subject of an active discussion. Stars are generally believed to be formed in dense molecular clouds and the transition from warm atomic hydrogen to cold molecular one is the most important step in this process, although the formation of stars directly from $\mathrm{HI}$ is also considered (Glover and Clark 2011). Nevertheless, the fact that the distribution of young stars in galaxies correlates precisely with $\mathrm{H}_{2}$ regions is beyond question (Leroy et al. 2008; Bigiel et al. 2011). Since direct observations of molecular clouds cause many difficulties, the cold $\mathrm{H}_{2}$ gas is observed predominantly by accompanying molecules, primarily $\mathrm{CO}$ (the $\mathrm{J}=1.0$ transition at $115 \mathrm{GHz}$ ), which take the second place in abundance in the interstellar medium after $\mathrm{H}_{2}$.

Determining the conversion factor $\chi$ between the CO intensity and the molecular hydrogen surface density is a separate problem. Observations of our Galaxy and its immediate neighborhood (see Bolatto et al. 2008; Leroy et al. 2011), where the molecular gas regions can be studied in more detail, play a crucial role here. There is reason to believe that $\chi$ is constant in a fairly wide range of gas densities from diffuse $\mathrm{H}_{2}$ to dense self-gravitating clouds (Liszt et al. 2010). However, it depends on metallicity, at least in the case of a strong heavy elements deficiency (Boselli et al. 2002; Leroy et al. 2011).

The radial distributions of the atomic and molecular gases differ greatly: whereas the azimuthally averaged HI surface density over a large extent changes only slightly along the radius and is $\sim 10 \mathrm{M}_{\odot} / \mathrm{pc}^{2}$ for normal galaxies for the inner disk regions, the $\mathrm{CO}$ emission is more strongly concentrated to the center. Investigating the $\mathrm{H}_{2} / \mathrm{HI}$ ratio and searching for the key parameters of the

*anastasya.kasparova@gmail.com interstellar medium responsible for the balance between these components, which is of crucial importance for understanding the mechanisms inducing the formation of stars and inhibiting this process, are of particular interest.

There are exist several theoretical models for the formation of molecular clouds (Elmegreen 1989; McKee and Krumholz 2010; Girichidis et al. 2011). For a wide range of parameters, these models are in good agreement with the well-known empirical relationship between the gas surface density ratio $\eta=\Sigma_{H_{2}} / \Sigma_{H I}$ and the equilibrium turbulent interstellar gas pressure $P$ changing along the radii of galaxies (Blitz and Rosolowsky 2006). The relationship exists despite the fact that the $\mathrm{H}_{2}$ distribution in galaxies has a complex structure: part of $\mathrm{H}_{2}$ is in a diffuse form and part is in giant molecular clouds, with the ratio of these components being not quite certain and may change with galactocentric distance (Rosolowsky and Blitz 2005). In the regions where the molecular gas in the form of self-gravitating clouds dominates, the dependence $\eta(P)$ is, in general, not obvious. The idea that the balance between $\mathrm{HI}$ and $\mathrm{H}_{2}$ depends not so much on the pressure as on the total surface density and metallicity of the interstellar gas (Krumholz et al. 2009) competes with the latter model. The amount of heavy elements controls the formation of $\mathrm{H}_{2}$ molecules, while the layer of interstellar gas shields them from the destructive action of ultraviolet radiation.

For normal galaxies, the dependences $\eta(P)$ and $\eta\left(\Sigma_{\text {gas }}\right)$ show an equally good correlation (Krumholz et al. 2009) and it is rather difficult to determine which of the physical mechanisms is decisive. Nevertheless, under special conditions, for example, in metal-poor dwarf galaxies, it becomes possible to check the validity of these models (Fumagalli et al. 2010). For such objects, the discrepancy between the dependences being discussed is significant, and the correlation $\eta\left(\Sigma_{\text {gas }}\right)$ probably better corresponds to the observed characteristics of the gas medium than $\eta(P)$. Nevertheless, the cloud formation models were calculated by Krumholz et al. (2009) by taking into account the complex mass transfer processes in the 


\begin{tabular}{|c|c|c|c|c|c|c|c|c|c|c|c|}
\hline & NGC & Iessier & $\begin{array}{c}D_{M 87} \\
\operatorname{deg}\end{array}$ & $\begin{array}{c}R_{25} \\
\operatorname{arcmin}\end{array}$ & type & $\begin{array}{c}V_{r} \\
\mathrm{~km} / \mathrm{s}\end{array}$ & $\begin{array}{c}\text { defici } \\
\mathrm{H}_{2}\end{array}$ & $\begin{array}{c}\text { iency } \\
\mathrm{HI}\end{array}$ & $\begin{array}{c}\text { refer } \\
\mathrm{H}_{2}\end{array}$ & $\begin{array}{c}\text { ences } \\
\mathrm{HI}\end{array}$ & $\mathrm{N}$ \\
\hline (1) & $(2)$ & (3) & (4) & $(5)$ & (6) & $(7)$ & (8) & (9) & $(10)$ & (11) & (12) \\
\hline 1 & 4254 & 99 & 3.6 & 2.69 & 5.2 & 2410 & -0.20 & 0.18 & {$[1]$} & {$[2]$} & I \\
\hline 2 & 4298 & & 3.2 & 1.48 & 5.2 & 1140 & & & {$[1]$} & {$[3]$} & III \\
\hline 3 & 4302 & & 3.1 & 2.45 & 5.4 & 1118 & & & [1] & {$[3]$} & III \\
\hline 4 & 4303 & 61 & 8.2 & 3.46 & 4.0 & 1570 & -0.08 & 0.13 & {$[1]$} & {$[2]$} & I \\
\hline 5 & 4321 & 100 & 3.9 & 3.01 & 4.1 & 1579 & 0.05 & 0.53 & {$[1]$} & {$[2]$} & I \\
\hline 6 & 4402 & & 1.4 & 1.77 & 3.2 & 119 & 0.02 & 0.73 & [1] & {$[2]$} & III \\
\hline 7 & 4419 & & 2.8 & 1.95 & 1.1 & -254 & 0.40 & 1.04 & [1] & {$[3]$} & II, III \\
\hline 8 & 4501 & 88 & 2.1 & 3.38 & 3.4 & 2280 & 0.14 & 0.51 & [1] & {$[2]$} & I \\
\hline 9 & 4535 & & 4.3 & 3.46 & 5.0 & 1958 & 0.02 & 0.35 & {$[1]$} & {$[2]$} & II \\
\hline 10 & 4536 & & 10.2 & 3.54 & 4.3 & 1807 & 0.18 & 0.29 & {$[1]$} & [3] & I \\
\hline 11 & 4548 & 91 & 2.4 & 2.63 & 3.1 & 486 & 0.84 & 0.70 & {$[1]$} & {$[2]$} & II \\
\hline 12 & 4567 & & 1.8 & 1.38 & 4.0 & 2265 & & & {$[1]$} & {$[3]$} & I \\
\hline 13 & 4568 & & 1.8 & 1.19 & 4.1 & 2255 & & & {$[1]$} & {$[3]$} & I \\
\hline 14 & 4569 & 90 & 1.7 & 4.56 & 2.4 & -233 & 0.23 & 1.13 & {$[1]$} & {$[2]$} & I \\
\hline 15 & 4579 & 58 & 1.8 & 2.51 & 2.8 & 1518 & 0.62 & 0.71 & {$[1]$} & {$[2]$} & II \\
\hline 16 & 4647 & & 3.2 & 1.41 & 5.2 & 1415 & & & {$[1]$} & {$[2]$} & I \\
\hline 17 & 4654 & & 3.3 & 2.51 & 5.9 & 1034 & -0.02 & 0.17 & {$[1]$} & {$[2]$} & I \\
\hline 18 & 4689 & & 4.3 & 2.29 & 4.7 & 1613 & 0.14 & 1.06 & {$[1]$} & {$[2]$} & I \\
\hline
\end{tabular}

TABLE I: Parameters of the Virgo cluster galaxies. Columns 8 and 9 give the references to the data on the molecular and atomic hydrogen profiles that we used: [1] —Chung et al. (2009a), [2]—Cayatte et al. (1994), [3] - Chung et al. (2009b).

medium but under a large number simplifications for a fairly simplegeometry and without allowance for the possible environmental effects.

In this paper, we focus our attention on spiral galaxies of the Virgo cluster distinguished by a great variety of relative $\mathrm{H}_{2}$ abundances. Checking the relations $\eta(P)$ and $\eta\left(\Sigma_{\text {gas }}\right)$ for these objects will show within the framework of which model the environmental effects on the interstellar medium can be described more properly and what additional physical parameters should be included in the description. Cluster galaxies are subjected both to the gravitational effect from other cluster members and to the influence of the intergalactic medium. As a result, a deficiency of atomic hydrogen is formed in galactic disks (Vollmer et al. 2001; Roediger 2009). However, the changes in HI abundance generally have no effect on the total $\mathrm{H}_{2}$ mass (Kenney and Young 1986, 1989), and only in rare cases does the environmental effects lead to a strong deficiency of both components (Fumagalli et al. 2009). In this paper, we will concentrate on studying the properties of the atomic and molecular interstellar medium in Virgo cluster galaxies. In our subsequent publications, we are planning to investigate in more detail the possible causes of the peculiarities of the relationship between these components.

The paper consists of three main sections: in Section 2, we discuss the technique used to numerically calculate the hydrostatic gas pressure in the disk midplane and present the results for the galaxies of our sample; in Section 3, we consider in detail the characteristics of the gas components of these objects; in Section 4, we discuss our results and the prospects for further studies.

\section{ESTIMATING THE GAS PRESSURE IN THE DISK MIDPLANE}

Blitz and Rosolowsky (2006) calculated the equilibrium gas pressure $\mathrm{P}$ in the disk midplane under considerable simplifications and without allowance for the gas self-gravity and the dark halo effect, which become significant on the periphery of galactic disks. However, precisely the outer regions of the gas disks affected most strongly by the environment are most interesting for our purposes. Therefore, here we use a more accurate self-consistent method and apply it to two samples: a sample of Virgo cluster galaxies and, for comparison, a sample of field galaxies. To derive the radial pressure profiles, we use the observed $\mathrm{HI}$ and $\mathrm{H}_{2}$ surface densities, stellar surface brightnesses, integrated color indices, and rotation curves for determining the stellar disk velocity dispersion. 


\begin{tabular}{ccccccccc}
\hline \hline \multicolumn{3}{c}{ NGC Messier } & \multicolumn{2}{c}{$R_{25}$} & \multicolumn{3}{c}{ type } & \multicolumn{2}{c}{ deficiency } \\
& & & $\mathrm{Mpc}$ & $\mathrm{kpc}$ & & $\mathrm{H}_{2}$ & $\mathrm{HI}$ \\
\hline$(1)$ & $(2)$ & $(3)$ & $(4)$ & $(5)$ & $(6)$ & $(7)$ & $(8)$ \\
\hline 1 & 628 & 74 & 7.3 & 10.4 & 5.2 & 0.19 & -0.01 \\
2 & 2841 & & 14.1 & 14.2 & 3.0 & & \\
3 & 3184 & & 11.1 & 11.9 & 6.0 & 0.11 & 0.32 \\
4 & 3198 & & 13.8 & 13.0 & 5.2 & & \\
5 & 3351 & 95 & 10.1 & 10.6 & 3.1 & 0.34 & 0.60 \\
6 & 3521 & & 10.7 & 12.9 & 4.0 & 0.19 & 0.06 \\
7 & 3627 & 66 & 9.3 & 13.9 & 3.1 & -0.11 & 0.81 \\
8 & 4736 & 94 & 4.3 & 5.3 & 2.4 & 0.78 & 0.68 \\
9 & 5055 & 63 & 10.1 & 17.4 & 4.0 & 0.04 & 0.12 \\
10 & 5194 & 51 & 8.4 & 27.4 & 4.0 & -0.36 & 0.12 \\
11 & 6946 & & 5.9 & 9.8 & 5.9 & -0.28 & -0.45 \\
12 & 7331 & & 14.7 & 19.6 & 3.9 & 0.14 & -0.04 \\
\hline \hline
\end{tabular}

TABLE II: Parameters of the field galaxies.

\subsection{The Pressure Estimation Technique}

In present-day works, the Poisson and hydrostatic equilibrium equations are simultaneously solved to estimate the gas pressure in the disk midplane. In this case, a number of simplifying assumptions are usually made. For example, Blitz and Rosolowsky $(2004,2006)$ calculated the pressure for infinite two-component disks (stars and gas) with a vertical scale height of the gas layer much smaller than that of the stellar disk and without allowance for the spheroidal components and gas self-gravity. Under these assumptions, they obtained an expression for the gas pressure:

$$
P=0.84\left(G \Sigma_{\text {star }}\right)^{0.5} \Sigma_{\text {gas }} \frac{v_{\text {gas }}}{h_{\text {star }}^{0.5}}
$$

where $G$ is the gravitational constant, $\Sigma_{\text {star }}$ and $\Sigma_{\text {gas }}$ are the stellar and gas surface densities, $v_{\text {gas }}$ is the turbulent gas velocity dispersion, and $h_{\text {star }}$ is the stellar scale height. The authors assumed that the last two parameters changed only slightly both with distance from the objects center and from galaxy to galaxy. In this approach, the pressure turns out to be a function of only the stellar and gas surface densities. However, as was justly pointed out by the authors themselves, when the gas becomes predominantly molecular (in the central regions of galaxies), the linear dependence of $\eta=\Sigma_{H_{2}} / \Sigma_{H I}$ on the pressure reflects the method of estimating $P$ than the physical relationship between the quantities being compared. As was shown by Kasparova and Zasov (2008), the simplifications listed above for an individual galaxy lead to a considerable underestimation of the pressure (up to $40 \%$ ) at distances greater than or of the order of $R_{25}$, where the gas self-gravity and the presence of a dark halo can play a significant role. However, for our goals, the disk peripheries are of the primary interest, because the influence of the intergalactic medium is most significant precisely on them. Therefore, here we use a more appropriate method of calculating the turbulent gas pressure.

We calculate the gas pressure from the gas volume density in the disk midplane under the assumption of a radius-independent turbulent velocity $v_{\text {gas }}$ but with allowance made for the gas selfgravity, the change in stellar disk thickness with radius, and the dark halo contribution to the galactic gravitational potential. The volume density was found through a self-consistent solution of the equations that describe the vertical structure of the stellar, atomic, and molecular disk components. This approach was proposed by Narayan and Jog (2002) for our Galaxy and was developed by Kasparova and Zasov (2008) and Abramova and Zasov (2011). Since the turbulent gas velocity does not change greatly from galaxy to galaxy, we take it to be 9 and $6 \mathrm{~km} / \mathrm{s}$ (in one coordinate) for the atomic and molecular gases, respectively. Note that the result is barely sensitive to the choice of $v_{\text {gas }}$ : if we underestimated this velocity, then this would lead to an overestimation of the resultant volume density of the component that would largely "quench" the effect of the error in the gas velocity dispersion on the resultant pressure (a twofold increase in $v_{\text {gas }}$ will cause $P$ to rise only by a factor of 1.5 rather than 2 , as follows from the simplified formula (1)).

The final expression for each galactic disk component derived from the Poisson and hydrostatic equilibrium equations is

$\frac{d^{2} \rho_{i}}{d z^{2}}=\frac{\rho_{i}}{\left\langle\left(v_{z}\right)_{i}^{2}\right\rangle}\left[-4 \pi G \sum_{i=1}^{3} \rho_{i}-\frac{\partial^{2} \phi_{d}}{\partial z^{2}}\right]+\frac{1}{\rho_{i}}\left(\frac{d \rho_{i}}{d z}\right)^{2}$,

where the term in square brackets corresponds to the potential of a three-component axisymmetric disk inside a halo, the index $i$ denotes one of the disk componentsthe stars, $\mathrm{HI}$, or $\mathrm{H}_{2}$, while $\phi_{d}$ corresponds to the potential of the dark halo that we assumed to be pseudo-isothermal. The system of equations for the stellar disk and the gas subsystems was solved numerically by the fourthorder Runge-Kutta method with boundary conditions in the disk midplane $z=0: \rho_{i}=\left(\rho_{0}\right)_{i}$ and $d \rho_{i} / d z=0$ (for more details, see Kasparova and Zasov 2008). We determined the stellar velocity dispersion from the rotation curve and the surface density of the disk under the assumption of its marginal stability. Note that for this method of calculation, the resultant stellar volume density, to a first approximation, depends only on the local epicyclic frequency but not on the surface density (see the Appendix in Abramova and Zasov (2011)). Another, the most popular method of estimating the stellar density, when the stellar disk thickness is constant (assuming the vertical and 
horizontal scale lengths of the stellar disks to be proportional), gives volume densities of the components that agree well with the previous method (Kasparova and Zasov 2008; Abramova and Zasov 2011). As a result, for a number of galaxies that both belong and do not belong to the Virgo cluster, we obtained radial volume density distributions for the gas components in the disk midplane proportional to the equilibrium turbulent pressure.

It is worth noting that using the azimuthal averaging in our calculations can introduce an uncertainty, because it is unknown how strongly the cluster environment affects the homogeneity of the gas components in azimuthal angle in the central disk regions. In prospect, it will not be out of place to investigate the influence of the cluster environment using two-dimensional distributions.

\subsection{Dependence of the Molecular Gas Fraction on the Gas Pressure}

To ascertain how the membership of a galaxy in a cluster affects the relationship between the molecular gas fraction and pressure, we calculated the radial gas pressure profiles for 18 Virgo cluster galaxies and, for comparison, 12 well-studied nearby spirals outside clusters (field galaxies from here on). The objects were chosen so that the observational data for them were as homogeneous as possible.

In Table 1, columns 2 and 3 give the NGC and Messier numbers of the Virgo cluster galaxies; column 4 - the projected distance to the Virgo cluster center (in degrees); column 5-the angular optical radius of the galaxy $R_{25}$; column 6 -the Hyper Leda morphological type; column 7 - the heliocentric velocity; columns 8 and 9-the references to the sources of $\mathrm{H}_{2}$ and $\mathrm{HI}$ data used: [1] — Chung et al. (2009a), [2] — Cayatte et al. (1994), [3] —Chung et al. (2009b); column 10 - the numbers of the subsamples to which we assigned the objects (they will be discussed below). We assume the distances to all cluster galaxies to be $16.1 \mathrm{Mpc}$ (Ferrarese et al. 1996). Table 2 provides data on the field galaxies.

To calculate the gas pressure, we used the radial distributions of the azimuthally averaged atomic and molecular hydrogen surface densities. For the cluster galaxies, we used the catalog of CO observations (Chung et al. 2009a) with a 45" resolution and the HI catalog by Cayatte et al. (1994) with the same resolution. For several objects absent in Cayatte et al. (1994), we took the data from Chung et al. (2009b) reduced to the same resolution. For the field galaxies, the data on the gas components were taken from Leroy et al. (2008). For all galaxies, the radial $\mathrm{H}_{2}$ profile was taken from the published data on ${ }^{12} C O(J=1-0)$ using the conversion factor $\chi=N_{H_{2}} / I_{C O}=2 \times 10^{20}$ $(K \cdot \mathrm{km} / \mathrm{s})^{-1} \mathrm{~cm}^{-2}$. We also used the observed ro- tation curves and the surface brightnesses of the stellar disks from Koopmann et al. (2001), Dicaire et al. (2008), Rubin et al. (1999), Heald et al. (2007), Sofue and Rubin (2001), Gavazzi et al. (2003), Nishiyama et al. (2001), Daigle et al. (2006) and Devereux et al. (1992). These were converted to the stellar surface density via the mass-to-light ratio corresponding to the integrated color index (Bell and de Jong 2001) in the infrared from NED. The central regions at $R<1$ kpc were not considered primarily because of the bulge effect disregarded in our models.

We plotted the molecular gas fraction $\eta$ against the total hydrostatic gas pressure $P=\rho_{H I} v_{H I}^{2}+$ $\rho_{H_{2}} v_{H_{2}}^{2}$ (in the approximation of a continuous medium) in the disk midplane (Fig. 1) for all of our galaxies.

In Fig. 1, the large scatter of curves relative to the Blitz-Rosolowsky linear dependence engages our attention. In contrast to most of the field galaxies, a significant fraction of the cluster members at the same gas pressure have a higher $\mathrm{H}_{2}$ fraction, especially on the periphery, than that following from the Blitz-Rosolowsky dependence. It may be caused by the fact that either we grossly underestimate the pressure acting on the interstellar medium and contributing to the phase transition to molecules or the cluster galaxies are characterized by different conditions determining the content of the molecular medium. It is worth noting that some of the field galaxies (NGC 2841, NGC 3351, and NGC 3627) also lie in the region of an enhanced molecular gas fraction. This probably reflects the peculiarities of their evolution, which requires a separate consideration.

The Virgo cluster galaxies can be arbitrarily divided into three subsamples with a different behavior of the dependence $\eta(P)$ (see the last column in Table 1). For clarity, each group in Fig. 1 is highlighted by the thick lines.

The first group (Fig. 1b) includes the galaxies whose behavior in the central regions is almost indistinguishable from that of the field galaxies, but there is a significant dispersion on the periphery and the molecular gas fraction is higher on average than that expected at a given pressure (or a pressure that is an order of magnitude lower at a given $\eta)$. Among the objects of the first group, there is a close pair, NGC $4567 /$ NGC 4568 , and this suggests that the observed scatter on the dependence $\eta(P)$ is not related to the local interaction between close galaxies.

We attribute the objects for which the molecular gas fraction is higher than that for single galaxies even in the central regions to the second group. It includes three galaxies: NGC 4419, NGC 4535, and NGC 4548. The dependences for them (Fig. 1c) lies much higher than for the field galaxies. Besides, there is reason to suggest that the galaxy NGC 4579 also belongs to this group, although, 


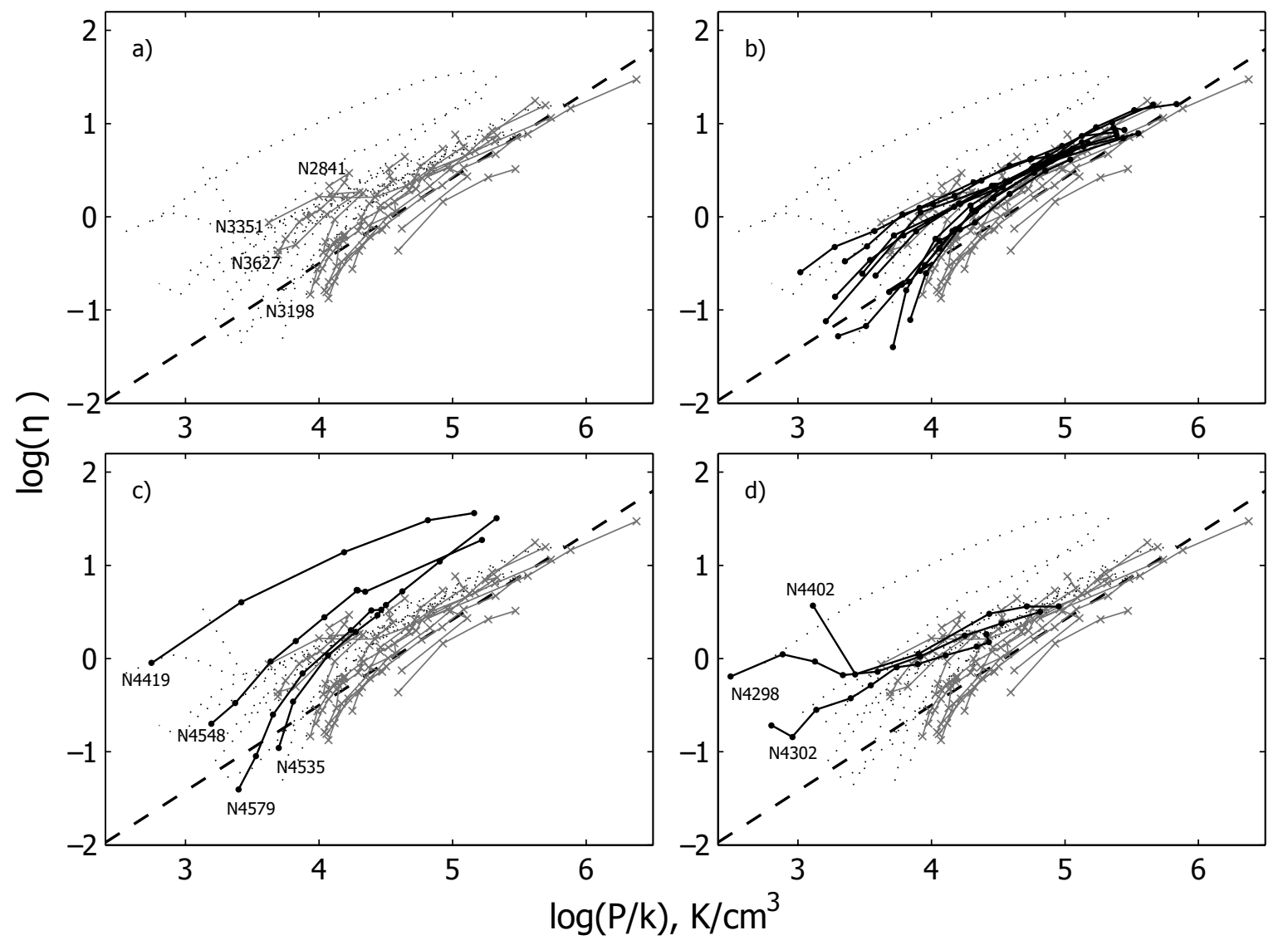

FIG. 1: Molecular hydrogen fraction $\eta$ versus turbulent gas pressure in the disk midplane for the field (a) and cluster (b, c, d) galaxies. On all plots, the gray lines with crosses indicate the field galaxies and the dotted lines indicate the Virgo cluster galaxies. The dashed straight line corresponds to the dependence $\eta \propto P^{0.92}$ (Blitz and Rosolowsky 2006). The cluster galaxies were divided into three groups described in the text. For clarity, each group is displayed on separate plots in comparison with the field galaxies: (b) for the Virgo cluster galaxies belonging to group I, (c) for group II, and (d) for group III.

according to the main source of $\mathrm{HI}$ observations that we use (for data homogeneity) (Cayatte et al. 1994), the profile in the central regions was not measured. Nevertheless, according to more recent data (Chung et al. 2009b), a dip is noticeable at the center of the $\Sigma_{H I}(R)$ distribution.

We place the galaxies NGC 4298, NGC 4402, and NGC 4302 in the third group. They are characterized by a considerable excess of molecular hydrogen (or a deficiency of atomic one) for a given pressure on the disk peripheryin fact, the anticorrelation region (Fig. 1d). Formally, we can also point out the galaxy NGC 4419 entering the second group by its anomalously high value of $\eta$ on the periphery. For three of these objects, $\eta$ is an order of magnitude higher than that typical of the field galaxies.

It is worth noting that the large scatter on the dependence $\eta(P)$ for the Virgo cluster galaxies cannot be explained by the pressure underestimation due to the inaccuracy of the approximate model. Besides, we have no reasons to suggest that our model is unsuitable precisely for the cluster galaxies, although we assumed the disks to be coplanar and axisymmetric, while the axial symmetry of the gas components of the cluster galaxies, indeed, can break but farther from the center-in the regions that we do not consider here, because there are no CO data for them. We also estimate the stellar velocity dispersion by assuming the stellar disks to be marginally stable. Generally, however, the stellar disks can have a reserve of stability, i.e., they can be thicker and more tenuous: the estimated gas volume density at the same surface density will then be lower than that for the marginal case and, hence, the gas pressure will be even lower. Thus, for overheated disks the deviation from the BlitzRosolowsky dependence only increases. 


\section{THE GAS COMPONENTS OF THE CLUSTER AND FIELD GALAXIES}

Let us consider the possible causes of the differences noted above for the field and cluster galaxies. An anomalous (higher than that observed in the field galaxies) ratio of the molecular and atomic hydrogen surface densities is possible, for example, when overestimating the number of $\mathrm{H}_{2}$ molecules (e.g., as a result of the overestimation of the conversion factor $\chi$ ) or due to the action of the intergalactic gas ram pressure "stripping" the atomic disk or if the environmental effects stimulate a more rapid transition of the atomic gas to molecules. Let us analyze these possibilities.

\subsection{The Conversion Factor}

The difference in gas metallicity that appeared due to a different star formation history (or, for example, because of the difference in the initial mass function of massive stars) could be responsible for the deviation of the conversion factor $\chi$ from the standard one for the cluster galaxies. For the Virgo cluster galaxies close to the cluster center, an enhanced abundance of heavy elements, on average, by $0.25-0.30$ dex relative to the solar one was actually pointed out (Shields et al. 1991; Skillman et al. 1996; Dors and Copetti 2006). For most of the galaxies, the relationship between metallicity and conversion factor $\chi \propto Z^{-1}$ proposed by Boselli et al. (2002) is most likely valid; hence a twofold excess of heavy elements can lead to an overestimation of $\chi$ approximately by a factor of 2 and, accordingly, to an overestimation of the $\mathrm{H}_{2}$ mass by a factor of 2 . For some of the galaxies, the dependence $\chi(Z)$ is probably stronger-for them, the overestimation of the amount of molecular gas can reach an order of magnitude (Magrini et al. 2011). However, even in this case, the scatter on the dependence $\eta(P)$ can be explained by an enhanced metallicity only for the galaxies of the first group, although it is worth noting that the anomaly in $\eta$ for them is observed only on the disk periphery, where, obviously, there are less heavy elements and, hence, the overestimation of the amount of $\mathrm{H}_{2}$ must be less significant than that in the central regions. However, for the galaxies of the second and third groups, the molecular gas fraction can exceed $\eta$ for normal-metallicity galaxies by two orders of magnitude (Figs. 1c, 1d) and this cannot be explained by a different conversion factor.

It is argued in a number of works that the conversion factor must depend on whether the molecular gas is in a diffuse state or in the form of giant self-gravitating clouds. Nevertheless, this question cannot yet be ultimately solved, although based on observations of the Galaxy, Liszt et al. (2010) recently showed that the generally not obvious but widespread assertion about universality of the conversion factor for forms of $\mathrm{H}_{2}$ is confirmed by observations.

\subsection{The Molecular Gas Fraction with Respect to the Total Gas Density}

As we have already mentioned in the Introduction, Krumholz et al. (2009) developed a model for the formation of molecular clouds whose key parameters are the total gas surface density and the amount of heavy elements in the interstellar medium. The higher the metallicity, the more efficient the formation of $\mathrm{H}_{2}$ molecules, and the cold molecular clouds are shielded from the destructive action of ultraviolet radiation as the thickness of the gas layer increases. For solar metallicity at $\Sigma_{\text {gas }}<10 \mathrm{M}_{\odot} / \mathrm{pc}^{2}$, the optical depth is insufficient to preserve the $\mathrm{H}_{2}$ molecules, and the fraction $\eta$ increases sharply.

In Fig. 2, $\eta$ is plotted against the gas surface density $\Sigma_{\text {gas }}=\Sigma_{H I}+\Sigma_{H_{2}}$ for our galaxies. The dashed lines indicate the model dependences from Krumholz et al. (2009) for various metallicities. Although the dependences are very similar in pattern to $\eta(P)$, the correlation breaks down noticeably on the plot of $\eta\left(\Sigma_{\text {gas }}\right)$ for some of the normal spirals along the radius (NGC 2841, NGC 3198, NGC 3351, and NGC 3627). Nevertheless, on the whole, the $\eta\left(\Sigma_{\text {gas }}\right)$ distributions for the field galaxies and the Virgo cluster galaxies of the first group, except the outer regions of the gas disks, agree with the model curves, especially if we consider a slightly enhanced metallicity for the cluster galaxies. However, for the galaxies of the second and third groups within the framework of the model by Krumholz et al., just as with the uncertainty of the conversion factor mentioned above, we will have to assume the metallicity to be more than an order of magnitude higher than the solar one, which is inconsistent with the available observations. It also follows from Fig. 2 that no distinct boundary surface density of about $10 \mathrm{M}_{\odot} / \mathrm{pc}^{2}$ below which the model by Krumholz et al. (2009) predicts a sharp decrease in $\eta$ is observed for the cluster galaxies.

Thus, it seems obvious that to explain the ratio of the gas components for the cluster galaxies, it is insufficient to consider such internal gas characteristics as the metallicity and surface density. The external factors related to the environmental effects on the balance between the gas components should also be taken into account.

\subsection{Peculiarities of the Radial $\mathrm{HI}$ and $\mathrm{H}_{2}$ Distributions}

The distributions of the molecular and atomic gas surface densities (Fig. 3) show that the most 

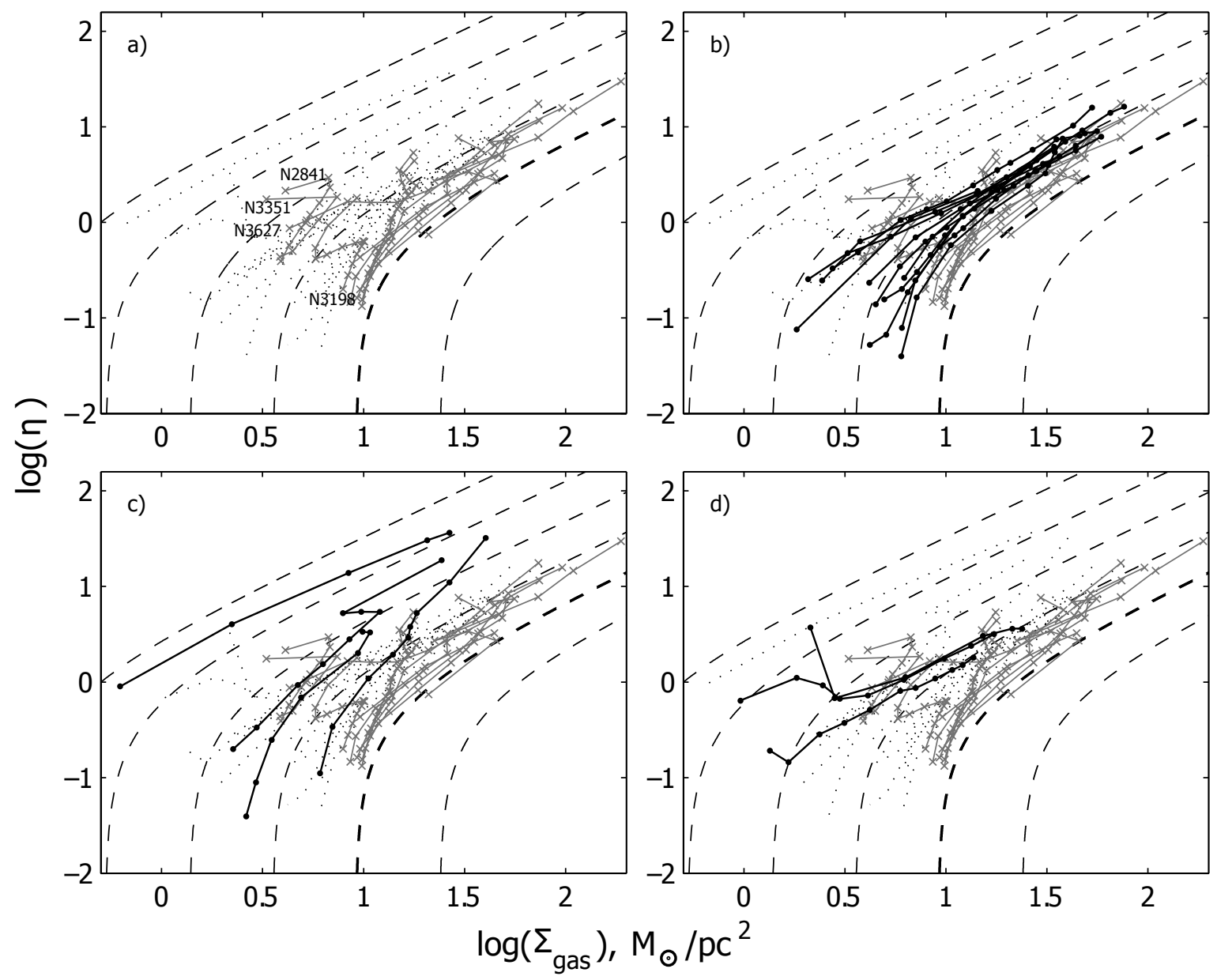

FIG. 2: Fraction of the molecular gas with respect to the atomic one versus total gas surface density. The dashed lines indicate the model dependences from Krumholz et al. (2009) for various metallicities: from right to left, the lines correspond to $\log \left(Z / Z_{\text {sun }}\right)$ equal to $-0.5,0,0.5,1,1.5$, and 2 . The remaining designations are the same as those in Fig. 1.

probable cause of the anomalously high $\eta$ for some of the cluster galaxies under consideration is a low atomic hydrogen abundance (at the center or on the periphery of the disks) rather than a high $\mathrm{H}_{2}$ abundance. A similar conclusion also follows from the volume density distributions of the gas components in the disk midplane that we derived. In comparison with the field galaxies, an HI deficiency is observed at all distances from the center for the objects of the second group (Fig. 3c) and to a greater or lesser extent on the periphery for the first and third groups (Figs. 3a, 3e). It is worth noting that, just as for the three field galaxies (NGC 2841, NGC 3351 NGC 3627) that we mentioned above, an HI deficiency is observed at various distances from the center due to the unusual behavior on the dependences $\eta(P))$ and $\eta\left(\Sigma_{\text {gas }}\right)$.

The ram pressure of the intergalactic medium, which sweeps out the diffuse gas, may be considered the most efficient HI loss mechanism (see Vollmer et al. 2001; Roediger 2009). Using a simple procedure, it can be verified whether the observed HI deficiency is enough to explain the anomalous ratio of the gas components $\eta$ for the cluster galaxies. Suppose that before the deficiency of atomic hydrogen emerged in the galaxies, they had a radial HI distribution typical for field galaxies of a given type. We will use the mean atomic hydrogen distributions for the galaxies of the corresponding morphological types (see Cayatte et al. 1994) by replacing the observed $\Sigma_{H I}(R)$ with them and leaving the observed $\mathrm{H}_{2}$ distribution unchanged. Subsequently, we will recalculate the equilibrium gas pressure corresponding to these "mixed" data. The black solid lines in Fig. 4 indicate the dependences $\eta(P)$ obtained in this way for the galaxies of different subgroups. For clarity, the dash-dotted lines indicate the initial dependences for the same groups of galaxies presented in Fig. 1. As follows from Fig. 4a, about half of the galaxies from the first group completely fell on the dependence typical of the field galaxies, i.e., the observed amount of molecular gas in them agrees well with that expected for the pressure $P$ 

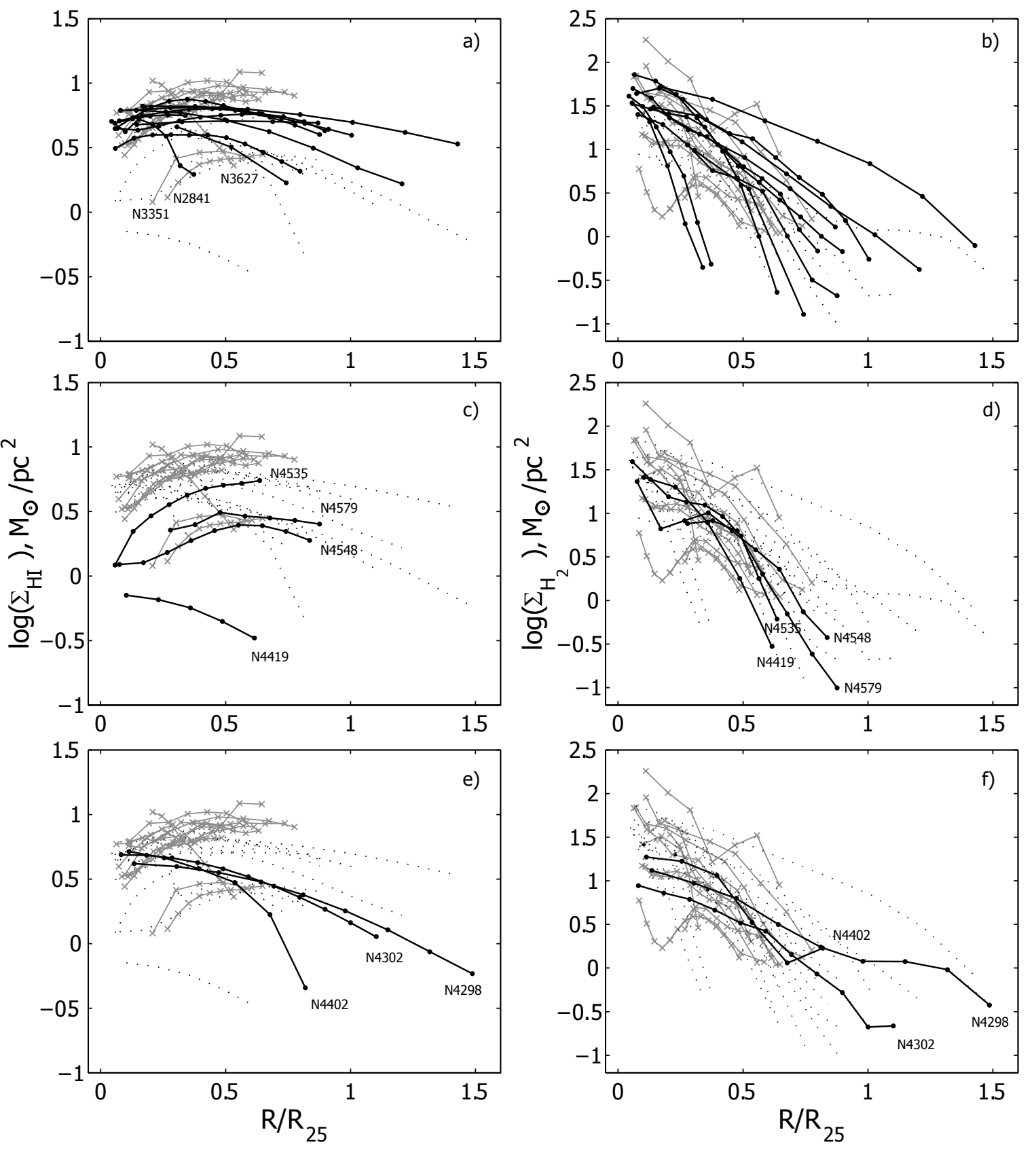

FIG. 3: Radial distributions of the atomic and molecular gas surface densities: (a) and (b) the atomic and molecular surface densities of the group I galaxies in comparison with those of the field galaxies; (c) and (d) the same for the group II galaxies; (e) and (f) for the group III galaxies. The designations are the same as those in Fig. 1.

existed before the loss of HI. For these galaxies, we clearly see a sharp decrease in $\eta$ on the disk periphery. This was obtained in our calculations, because the gas self-gravity and the dark halo effect were taken into account. This peculiarity agrees well with the model by Krumholz et al. (2009).

However, on the disk periphery for the rest of the galaxies from the first and third groups and for the central regions of the galaxies from the second group, the sweepout of HI explains only partly the high value of $\eta$ (Figs. 4b, 4c, and it becomes necessary to assume an additional transition from $\mathrm{HI}$ to $\mathrm{H}_{2}$ due to some factor that is most likely related to the environmental effects.

\section{CONCLUSIONS}

In this paper, we set the goal of investigating the atomic-to-molecular gas ratio in galaxies that 

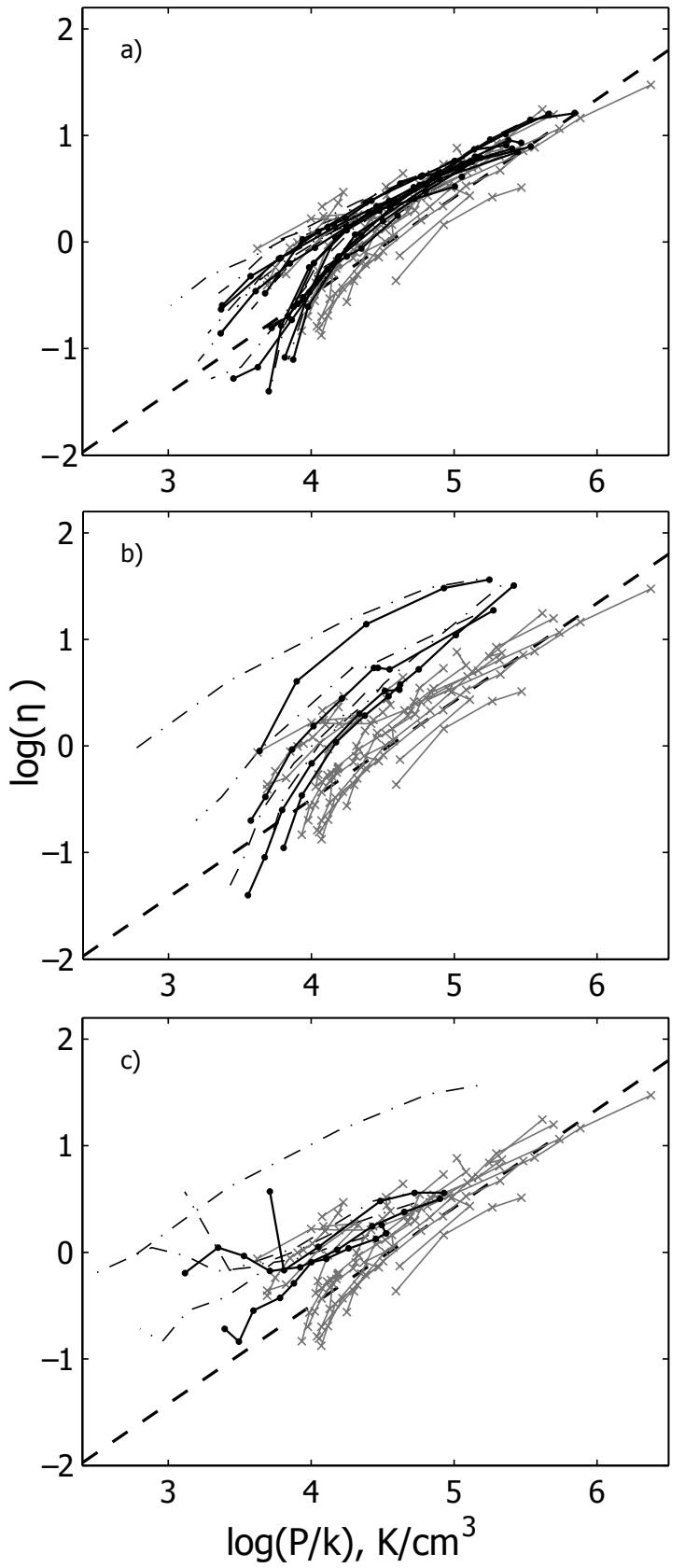

FIG. 4: Dependence of the molecular hydrogen fraction with respect to atomic hydrogen on the turbulent gas pressure in the disk midplane calculated for the model, average for a given morphological type, $\Sigma_{H I}$ distribution taken from Cayatte et al. (1994). For comparison, the dash-dotted line indicates the dependences for the galaxies of the same subgroups but with the original, observed atomic gas surface densities presented in Fig. 1.

enter and do not enter the Virgo cluster. The balance between the gas components of the galaxies can change under the action of the intergalactic medium, and the physics of one of the key star formation stages, the transition from a warm atomic medium to cold molecular clouds, can be traced by studying the peculiarities of the gas content.

To reveal the key characteristics of the interstellar medium establishing a certain ratio of the $\mathrm{HI}$ and $\mathrm{H}_{2}$ components, we compared two approaches: the dependence of the molecular gas fraction $\eta$ predominantly on the equilibrium hydrostatic turbulent pressure (Blitz and Rosolowsky 2006) or on the metallicity and total gas surface density (Krumholz et al. 2008, 2009; McKee and Krumholz 2010). The deviations from these correlations must be indicative either of an imbalance between the components or shortcomings of the equilibrium models. Both approaches satisfactorily explain the observed ratios of $\mathrm{HI}$ to $\mathrm{H}_{2}$ for the field galaxies, but there is a significant scatter in both cases for the cluster galaxies (Figs. 1 and 2). On the whole, the correlation $\eta(P)$ turned out to be slightly closer than $\eta\left(\Sigma_{\text {gas }}\right)$. This is particularly noticeable in some segments of the anticorrelation in the $\eta\left(\Sigma_{\text {gas }}\right)$ distribution that take place even for noninteracting galaxies. Nevertheless, the significant scatter for the cluster members compared to the field galaxies on both dependences requires explanations. For convenience, we divided the sample into three formal groups differing in behavior on the dependences under discussion (see Section 2.2 ) by assuming that based on these features, we can distinguish the various effects of the intergalactic medium on the gas of the cluster galaxies.

In Section 3.3, we showed that the high molecular gas fraction for the cluster galaxies could not be entirely explained by the stripping of HI. However, for most of the objects from the first group, the scatter on the dependence $\eta(P)$ can be explained almost entirely by a deficiency of atomic hydrogen at a normal $\mathrm{H}_{2}$ abundance. The remaining excess of $\eta$ above the expected values for several galaxies of the first group after allowance for the HI deficiency (Fig. 4a) can be explained by a lower conversion factor $\chi$ for the cluster galaxies (Magrini et al. 2011). For the galaxies of the second group, we should assume an excess $\mathrm{H}_{2}$ abundance for a given $P$ at least in the central regions, because even if the galaxy had no HI deficiency, the deviation from the dependence holding for the field galaxies cannot be removed (see Fig. 4b). These galaxies are characterized by a significant deficiency of both atomic and molecular gases. In contrast, the objects of the third group have an unusually high $\mathrm{H}_{2}$ fraction primarily not at the center but on the periphery of the disks (Fig. 4c).

Thus, under the assumption made by Blitz and Rosolowsky (2006) about the key importance of the interstellar gas pressure, we can entirely explain the balance between the atomic and molecular components of the gas medium for most of the field galaxies and, with allowance made for the deficiency of HI, for the first group of cluster galaxies. However, some disregarded factor related to the evolution of galaxies in a cluster and leading 
to growth of the molecular fraction in the central regions and on the periphery of the disks, respectively, obviously acts on the objects of the second and third groups. We will devote the next paper to this subject matter.

The following question also remains open: why does not the molecular gas density decrease, for example, due to star formation or the destruction of molecular clouds by ultraviolet radiation after the "removal" of HI from the galaxy or after the transition of the bulk of $\mathrm{HI}$ to $\mathrm{H}_{2}$ (it does not matter through what processes)? A possible way out of this situation would be a long life of molecular clouds: to be more precise, the cloud destruction time must be longer than the time elapsed after the decrease in the bulk of HI.

According to the model by Krumholz et al.,molecular clouds at solar metallicity can exist in considerable quantities only at a total gas surface density greater than $10 \mathrm{M}_{\odot} / \mathrm{pc}^{2}$. We see from Fig. 2 that this is not the case for many of the Virgo cluster galaxies, and $\eta$ can be great even at a lower gas density. To explain the behavior of most of these galaxies in terms of the model by Krumholz et al. (2009), it should be assumed that the metallicity for them is an order of magnitude higher than that for the field galaxies. However, observations show a difference only by a factor of 2 or 3 (Shields et al. 1991; Skillman et al. 1996; Dors and Copetti 2006). Nevertheless, we see good agreement with the model by Krumholz et al. for the field galaxies and the first group of cluster galaxies. In this model, the galaxies of the first group lie, on average, slightly higher than the field galaxies on the $\eta\left(\Sigma_{\text {gas }}\right)$ diagram by a value corresponding to a metallicity exceeding the solar one approximately by $0.3 \mathrm{dex}$ (Fig. 2b). However, the behavior of the galaxies from the second and third groups on the dependence $\eta\left(\Sigma_{\text {gas }}\right)$ cannot be explained in such a way, and the additional parameters reflecting the environmental effects on the molecular gas fraction should be included in the description for these objects.

\section{Acknowledgments.}

I wish to thank A.V. Zasov for his help in choosing the direction of research, productive scientific discussions, and valuable remarks. This work was in part supported by a grant from the President of Russian Federation for the State Support of Young Russian PhDs (MK 73.211.2) and the grant RFBR 11-02-12247-ofi-m-2011.

\section{REFERENCES}

1. O. V. Abramova and A. V. Zasov, Astron. Rep. 55, 202 (2011).

2. E. F. Bell and R. S. de Jong, Astrophys. J. 550, 212 (2001).
3. F. Bigiel, A. K. Leroy, F. Walter et al., Astrophys. J. 730, L13 (2011).

4. L. Blitz and E. Rosolowsky, Astrophys. J. 612, L29 (2004).

5. L. Blitz and E. Rosolowsky, Astrophys. J. 650, 933 (2006).

6. A. D. Bolatto, A. K. Leroy, E. Rosolowsky et al., Astrophys. J. 686, 948 (2008).

7. A. Boselli, J. Lequeux, and G. Gavazzi, Astron. Astrophys. 384, 33 (2002).

8. V. Cayatte, C. Kotanyi, C. Balkowski et al., Astron. J. 107, 1003 (1994).

9. E. J. Chung, M.-H. Rhee, H. Kim et al., Astrophys. J. Suppl. Ser. 184, 199 (2009a).

10. A. Chung, J. H. van Gorkom, J. D. P. Kenney et al., Astron. J. 138, 1741 (2009b).

11. O. Daigle, C. Carignan, P. Amram et al., Mon. Not. R. Astron. Soc. 367, 469 (2006).

12. N. A. Devereux, J. D. Kenney, and J. S. Young, Astron. J. 103, 784 (1992).

13. I. Dicaire, C. Carignan, P. Amram et al., Mon. Not. R. Astron. Soc. 385, 553 (2008).

14. O. L. Dors and M. V. F. Copetti, Astron. Astrophys. 452, 473 (2006).

15. B. G. Elmegreen, Astrophys. J. 338, 178 (1989).

16. L. Ferrarese, W. L. Freedman, R. J. Hill et al., Astrophys. J. 464, 568 (1996).

17. M. Fumagalli, M. R. Krumholz, J. X. Prochaska et al., Astrophys. J. 697, 1811 (2009).

18. M. Fumagalli, M. R. Krumholz, and L. K. Hunt, Astrophys. J. 722, 919 (2010).

19. G. Gavazzi, A. Boselli, A. Donati et al., Astron. Astrophys. 400, 451 (2003).

20. P. Girichidis, C. Federrath, R. Banerjee et al., Mon. Not. R. Astron. Soc. 413, 2741 (2011).

21. S. C. O. Glover and P. C. Clark, arXiv: 1105.3073 (2011).

22. G. H. Heald, R. J. Rand, R. A. Benjamin et al., Astrophys. J. 663, 933 (2007).

23. A. V. Kasparova and A. V. Zasov, Astron. Lett. 34, 152 (2008).

24. J. D. Kenney and J. S. Young, Astrophys. J. 301, L13 (1986).

25. J. D. Kenney and J. S. Young, Astrophys. J. 344, 171 (1989).

26. R. A. Koopmann, J. D. P. Kenney, and J. Young, Astrophys. J. Suppl. Ser. 135, 125 (2001). 27. M. R. Krumholz, C. F. McKee, and J. Tumlinson, Astrophys. J. 689, 865 (2008).

28. M. R. Krumholz, C. F. McKee, and J. Tumlinson, Astrophys. J. 693, 216 (2009).

29. A. K. Leroy, A. Bolatto, K. Gordon et al., Astrophys. J. 737, 12 (2011).

30. A. K. Leroy, F.Walter, E. Brinks et al., Astron. J. 136, 2782 (2008).

31. H. S. Liszt, J. Pety, and R. Lucas, Astron. Astrophys. 518, A45 (2010).

32. L. Magrini, S. Bianchi, E. Corbelli et al., Astron. Astrophys. 535, 13 (2011). 
33. C. F. McKee and M. R. Krumholz, Astrophys. J. 709, 308 (2010).

34. C. A. Narayan and C. J. Jog, Astron. Astrophys. 394, 89 (2002).

35. K. Nishiyama, N. Nakai, and N. Kuno, Publ. Astron. Soc. Jpn. 53, 757 (2001).

36. E. Roediger, Astron. Nachr. 330, 888 (2009).

37. E. Rosolowsky and L. Blitz, Astrophys. J. 623, 826 (2005).

38. V. C. Rubin, A. H. Waterman, and J. D. P. Kenney, Astron. J. 118, 236 (1999).
39. G. A. Shields, E. D. Skillman, and R. C. Kennicutt, Astrophys. J. 371, 82 (1991).

40. E. D. Skillman, R. C. Kennicutt, G. A. Shields et al., Astrophys. J. 462, 147 (1996).

41. Y. Sofue and V. Rubin, Ann. Rev. Astron. Astrophys. 39, 137 (2001).

42. B. Vollmer, V. Cayatte, C. Balkowski et al., Astrophys. J. 561, 708 (2001).

Translated by V. Astakhov 